\title{
67P/Churyumov-Gerasimenko activity evolution during its last perihelion before the Rosetta encounter
}

\author{
L. M. Lara ${ }^{1}$, Z.-Y. Lin $^{1}$, R. Rodrigo ${ }^{1}$, and W.-H. Ip $^{2}$ \\ 1 Instituto de Astrofísica de Andalucía (CSIC), Glorieta de la Astronomía s/n, 18008 Granada, Spain \\ e-mail: lara@iaa.csic.es \\ 2 Institute of Astronomy, National Central University, Taiwan \\ e-mail: wingip@astro.ncu.edu.tw
}

Received 2 August 2010 / Accepted 23 September 2010

\section{ABSTRACT}

\begin{abstract}
Context. The comet 67P/Churyumov-Gerasimenko, target of the Rosetta Mission (ESA) was monitored from January to April 2009 during its last perihelion passage before the Rosetta spacecraft encounters the comet nucleus in May 2014. Photometric data were obtained only from January 25 to March 19, 2009 and they were used to monitor the comet gas and dust activity. Non-photometric data are considered for analysing the evolution of the dust coma morphology.

Aims. The goal of the campaign was to characterize the comet activity evolution as it approaches the Sun. We aimed to assess gas and dust production rates shortly before perihelion and after perihelion, as well as to follow the evolution of the dust coma morphology during this passage.

Methods. Long-slit spectra and optical broadband images were acquired with the instrument CAFOS mounted at the 2.2. m telescope at Calar Alto Observatory (CSIC-MPG), and with the camera at the 1-m telescope of the Lulin Optical Observatory in Taiwan. We investigated the evolution of the dust coma morphology from the $R$ Johnson images with image enhancing techniques. When possible, we studied the dust and gas production rate, the radial profiles of the dust brightness azimuthally averaged and in the Sun-antisunward direction, and the Sun-antisunward profiles of the $\mathrm{CN}, \mathrm{C}_{2}, \mathrm{C}_{3}$, and $\mathrm{NH}_{2}$ column densities.

Results. The morphological analysis of the dust coma reveals that structures indeed exist. Aside from the dust tail in the SW direction, four more small-scaled structures have been detected by using the adaptive Laplacian filtering, the radial renormalization and the Sekanina-Larson method. These structures are also confirmed by the distortion of the isophotes at the same position angles (PA). During January and February, a faint structure could be seen at PA $330^{\circ}$, whereas this feature seems to have disappeared after perihelion. Additionally, the broad structure at PA $\sim 45^{\circ}$ seemed to split into two narrower ones on February 26 , to become only a considerably fainter one at PA $\sim 75^{\circ}$ during March. The Af $\rho$ values show considerable scatter in the few days we could measure them. There is a clear increase when the comet approaches the perihelion, however it is not possible to conclude where the peak dust activity is reached when the comet is at or beyond the closest distance to the Sun. The $\mathrm{CN}, \mathrm{C}_{2}, \mathrm{C}_{3}$, and $\mathrm{NH}_{2}$ production rates, $Q$, have been obtained at $r_{\mathrm{h}} 1.25 \mathrm{AU}$. These $Q$ 's are very similar to the ones derived from previous passages around perihelion. The decrease in the perihelion distance in 2009 (1.24 AU in 2009 vs. $1.30 \mathrm{AU}$ in 1982 and 1995) has not induced a noticeable increase either in the gas production rates or in the dust production rates, as measured from the Af $\rho$ parameter. The azimuthally averaged surface brightness profiles of the continuum from the broad band images can be well fitted with $-1.92 \leq m \leq-1.35$ in $\log B-\log \rho$ representation for $\rho \leq 100000 \mathrm{~km}$ projected cometocentric distance from the $R$ broadband images. On the other hand, when the fit is done in the sun-antisunward direction from the long-slit spectra at $\rho \leq 25000 \mathrm{~km}$, the slope $m \approx-1$.
\end{abstract}

Key words. comets: general - comets: individual: 67P/Churyumov-Gerasimenko

\section{Introduction}

On March 2, 2004, the ESA mission Rosetta was launched to explore the comet 67P/Churyumov-Gerasimenko (67P/C-G hereofafter) from May 2014 until December 2014, i.e. the nominal mission duration. The mission target was discovered in 1969 after its perihelion at 1.28 AU, although the comet had several perturbations by Jupiter (1840 and 1959), which have moved the perihelion distance from 2.7 AU prior to 1959 to the current values.

The available data for $67 \mathrm{P} / \mathrm{C}-\mathrm{G}$ is very scarce, because only a few apparitions have provided the ground-based observers with good visibility conditions and a good resulting dataset. There are reported observations during the 1982/83, 1995/96 and 2002/03 perihelion passages (Osip et al. 1992; Weiler et al. 2004; Lara et al. 2005; Schleicher 2006; Ferrín 2010). Results given in Osip et al. (1992) for the 1982/83 apparition conclude that comet $67 \mathrm{P} / \mathrm{C}-\mathrm{G}$ shows a significant asymmetry in both the dust and the gas production rates, with peak productivity occurring in the month after perihelion, and the measured dust-to-gas ratio was considerably high, even higher than for 1P/Halley. During the 1995/96 apparition, Schleicher (2006) obtained data during the pre-perihelion phase and during a few days after the perihelion passage itself. The time range was thus -61 to +118 days from perihelion such that the asymmetry already pointed out by Osip et al. (1992) could be analysed during one more passage. Also, Weiler et al. (2004) analysed spectrophotometric observations of 67P/C-G about 20 days after the 1995/96 perihelion passages, whereas Weiler et al. (2004) and Lara et al. (2005) also studied on the morphological analysis of the dust coma of the comet during the 2003 post-perihelion at $r_{\mathrm{h}}=2.36-2.78$. On one hand, Lara et al. (2005) report on dust structures in the inner coma at position angles of $295^{\circ}$ and $195^{\circ}$, as well as on a long spike in anti-tail direction reaching $230000 \mathrm{~km}$ identified as a 
Table 1. Log of photometric observations and results from $R$ broadband images.

\begin{tabular}{cccccccccc}
\hline \hline $\begin{array}{c}\text { Date } \\
2009\end{array}$ & $\begin{array}{c}r_{\mathrm{h}} \\
(\mathrm{AU})\end{array}$ & $\begin{array}{c}\Delta \\
(\mathrm{AU})\end{array}$ & $\begin{array}{c}\Delta T^{a} \\
(\mathrm{days})\end{array}$ & $\begin{array}{c}\mathrm{PA} \\
(\mathrm{deg})\end{array}$ & $\begin{array}{c}\alpha \\
(\mathrm{deg})\end{array}$ & $\begin{array}{c}\mathrm{Af} \rho_{10000 \mathrm{~km}} \\
(\mathrm{~cm})\end{array}$ & $m_{1}$ & $m_{2}$ & Location $^{b}$ \\
\hline January 25 & 1.314 & 1.671 & -34 & 67.8 & 36.1 & 245 & -1.47 & -1.37 & LOT \\
January 30 & 1.294 & 1.670 & -29 & 67.5 & 36.1 & 252 & -1.49 & -1.38 & LOT \\
January 31 & 1.291 & 1.669 & -28 & 67.4 & 36.1 & 250 & -1.49 & -1.49 & LOT \\
February 23 & 1.247 & 1.678 & -5 & 67.5 & 35.9 & 344 & -1.92 & -1.22 & LOT \\
February 25 & 1.247 & 1.680 & -3 & 67.5 & 35.8 & 257 & -1.45 & -1.18 & LOT \\
February 26 & 1.246 & 1.681 & -2 & 67.5 & 35.8 & 373 & -1.54 & -1.18 & LOT \\
March 12 & 1.256 & 1.709 & 12 & 69.2 & 35.1 & 426 & -1.58 & -1.09 & LOT \\
March 19 & 1.269 & 1.732 & 19 & 70.4 & 34.6 & 383 & -1.35 & -1.11 & CA \\
\hline
\end{tabular}

Notes. $r_{\mathrm{h}}$ and $\Delta$ are the heliocentric and geocentric distance of the component during the observations, respectively. PA is the position angle of the extended Sun-target radius vector. $\alpha$ is the phase angle Sun-comet-observer. Af $\rho$ values are derived for photometric circular aperture with radius $10000 \mathrm{~km}$ from the comet's nucleus. Errors on Af $\rho$ are of the order of $10 \%$ and are mainly due to absolute flux calibration errors. $m_{1}$ : slope of the linear fit $\log B$ vs. $\log \rho$ for $3.5 \leq \log \rho \leq 5$. Standard deviation of these fits is always lower than 5\%. $m_{2}:$ slope of the linear fit $\log B$ vs. $\log \rho$ for $3.5 \leq \log \rho \leq 4.4$. Standard deviation of these fits is always lower than $5 \%$. ${ }^{(a)}$ Days from perihelion; ${ }^{(b)}$ LOT stands for Lulin Optical Telescope (Taiwan) and CA for Calar Alto Observatory (Spain).

neck-line. On the other hand, Weiler et al. (2004) analysed the dust and gas production rate three weeks after the comet passed perihelion on Jan. 17, 1996 and found the same asymmetry as Osip et al. (1992). As a summary, from the data acquired during the last 3 Sun passages, the dust coma of comet 67P/C-G has displayed structures on large and small scales, whereas the gas and dust production rates peaked at about 1 month after perihelion.

The 2009 apparition has not had good visibility for groundbased observers, and usable data were only acquired during few hours in a short interval of time near perihelion, which took place on February 28, 2009. In this paper, we present non-photometric (for coma morphology description), photometric, and spectrophotometric measurements (both for deriving dust and gas production rates, respectively) from the end of January to mid-April 2009. The photometric observations monitor the comet from 1.314 AU pre-perihelion to 1.269 postperihelion (i.e. -34 to +19 days to perihelion), whereas the nonphotometric data follow the comet activity up to $r_{\mathrm{h}}=1.373 \mathrm{AU}$ post-perihelion on April 16, 2009. With this dataset, we have aimed at a global characterization of the Rosetta mission target during its last visit to the inner Solar System before the Rosetta spacecraft encounters the comet nucleus in May 2014.

\section{Observations and data reduction}

The comet was monitored from the Calar Alto Observatory (CSIC-MPG, near Almería, Spain) from February 22 until April 16, 2009, and from the Lulin Observatory (National Central University, Taiwan) from January 25 until March 22, 2009. Data from both observatories represents a total of $23 \mathrm{ob}-$ serving nights, in which broad-band images and spectroscopic measurements were acquired. However, only $\sim 30 \%$ of the planned data were obtained given the bad weather conditions at Calar Alto Observatory during the first semester of 2009, and not very good target visibility from Lulin Observatory. More concisely, broadband images acquired at either the Calar Alto observatory or Lulin Optical Telescope under non-photometric conditions have only been considered for an indepth morphological analysis (some of them shown and described here), which will be published in a forthcoming paper (Vincent et al. 2011, in prep.). All comet observations were done in service mode at both observatories with telescope tracking at the comet's proper motion. The typical mean seeing was $\sim 1.0-1.2^{\prime \prime}$ at both observatories.
Imaging. Imaging data were obtained with the central $1 \mathrm{k} \times$ $1 \mathrm{k}$ pixels of the instrument CAFOS (pixel size: 0.'53, FOV $\left.9^{\prime} \times 9^{\prime}\right)$ mounted at the $2.2 \mathrm{~m}$ telescope. The comet was imaged with Johnson $R$ broadband filter by acquiring consecutive series of 5 to 10 images. A considerable data set was also acquired with a CCD at the $1 \mathrm{~m}$ optical telescope at the Lulin Observatory, LOT, $\left(1340 \times 1300\right.$, pixel size $0.515^{\prime \prime}$, FOV $\left.11.0^{\prime} \times 11.2^{\prime}\right)$. The comet was imaged with $B, V, R$, and $I$ Asahi filters Appropriate bias, darks and flat field frames were also taken each night, and the usual data reduction was made. If photometric conditions prevailed, photometric stars were observed at an airmass similar to the comet observations for absolute flux calibration. Table 1 only lists the details of the imaging observations acquired during photometric conditions.

Spectroscopy. The spectroscopic measurements were done on February 22-24, and March 19, 2009. We used CAFOS with grism B200 and B400 (see http://www.caha.es/alises/ cafos/cafos 22 .pdf) providing us with an observable spectral range between 320 and $880 \mathrm{~nm}$ and a wavelength scale of $0.475 \mathrm{~nm}$ per pixel, and between 280 and $1000 \mathrm{~nm}$ with a wavelength scale of $0.97 \mathrm{~nm}$ per pixel, respectively. The slit of the spectrograph is oriented along the Sun-comet direction, as projected on the plane of the sky, giving dust and gas radial profiles in the Sun-antisunward direction. For absolute calibration, observations of appropriate spectrophotometric standard stars were acquired. For the comet observations, the slit width is $2^{\prime \prime}$, whereas the usable selected length is $10.6^{\prime}$, which provides us with radial profiles along selected directions up to cometocentric projected distances of $\geq 10^{5} \mathrm{~km}$. The spectrophotometric standard star was observed with a width of $5^{\prime \prime}$ and the same slit length.

Since the gas emission of $67 \mathrm{P} /$ Churyumov-Gerasimenko does not cover the entire length of the slit, it is possible to extract the sky contamination directly from the edges of the frame. More details on the images and spectra reduction and calibration can be found in Lara et al. (2001), Bertini et al. (2009) and Lin et al. (2009).

The images, regardless the sky conditions, have been used to study (i) the dust coma morphology by applying a Laplace filtering technique (Boehnhardt \& Birkle 1994), the radial renormalization, and the Sekanina-Larson method (Larson \& Sekanina 1984); (ii) the azimuthally averaged profile of surface brightnesses, whereas in photometric sky conditions, the images have 

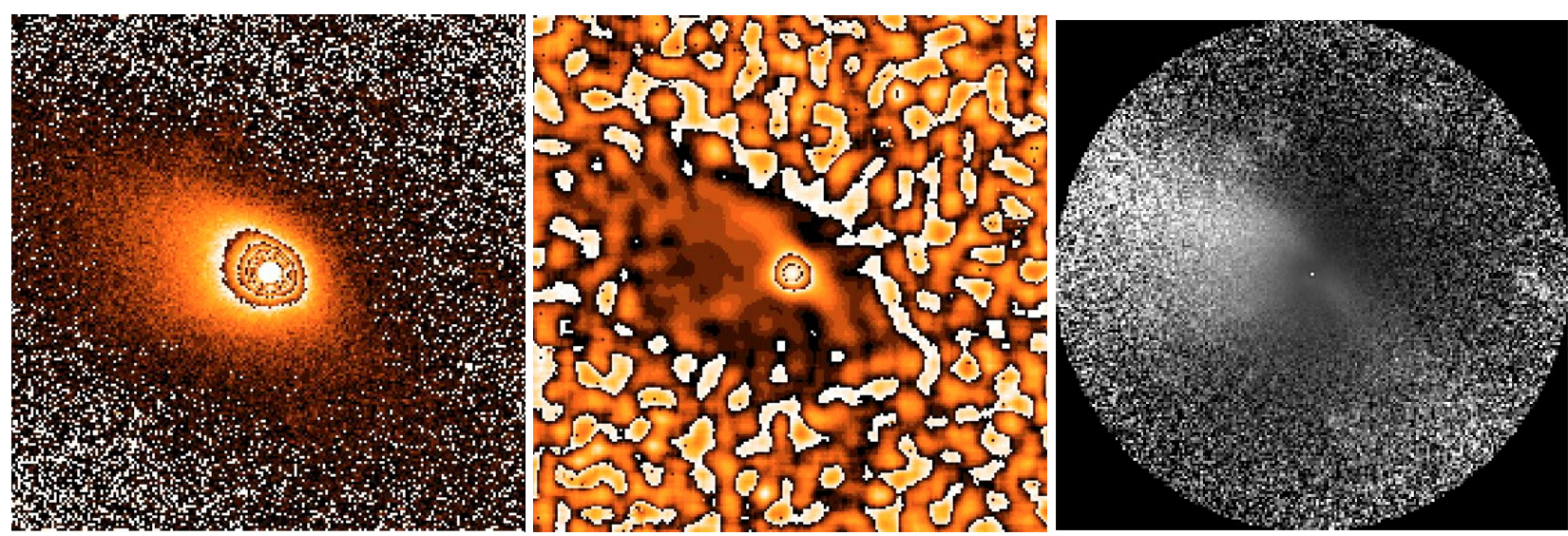

Fig. 1. Image of 67P/Churyumov-Gerasimenko on January 30, 2009, acquired with $R$ Johnson filter: a) corrected of flat, bias, and sky background, b) adaptive Laplace filtered, and c) enhanced by the radial renormalization technique. North is up and east to the left. The FOV is $103^{\prime \prime} \times 103^{\prime \prime}$, meaning $125000 \times 125000 \mathrm{~km}$ at the comet distance.
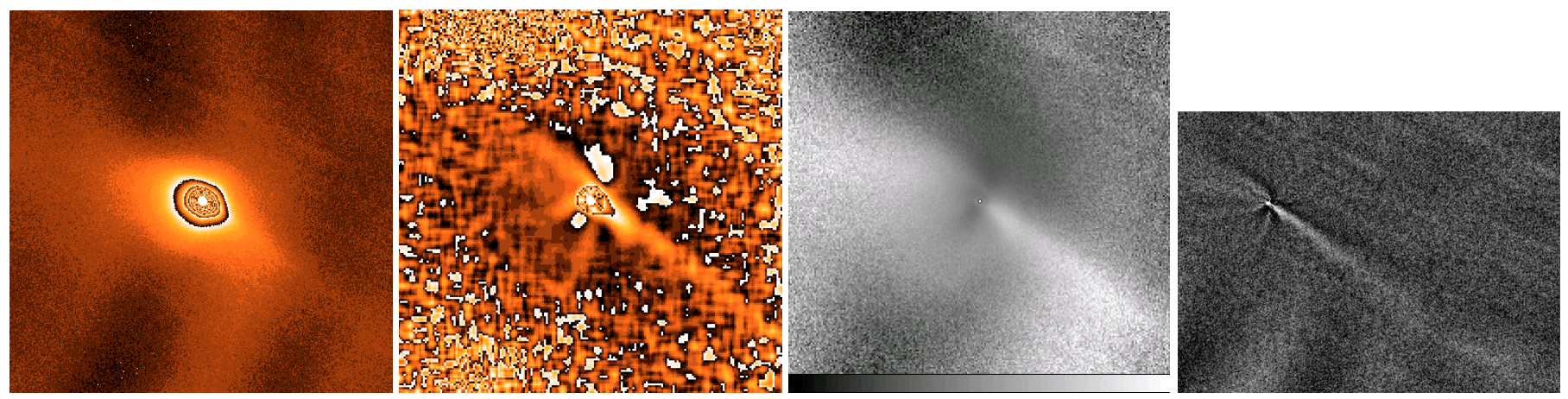

Fig. 2. Image of 67P/Churyumov-Gerasimenko on February 18, 2009, acquired with $R$ Johnson filter. From left to right: corrected of flat, bias, and sky background; Laplace filtered; radially renormalized; and enhanced by the Sekanina-Larson method. North is up and east to the left. The FOV is $212^{\prime \prime} \times 212^{\prime \prime}(192000 \times 192000 \mathrm{~km}), 106^{\prime \prime} \times 106^{\prime \prime}, 106^{\prime \prime} \times 106^{\prime \prime}(96000 \times 96000 \mathrm{~km})$, and $208^{\prime \prime} \times 158^{\prime \prime}(189000 \times 143000 \mathrm{~km})$. The isophotes in the observed image clearly indicate the presence of structures in the inner coma, which are nicely enhanced in the Laplace filtered image, as well as in the resulting frame of the Sekanina-Larson method. However, the radially renormalized image is most suitable for detecting broad and large-scale structures.

been used for analysing the behaviour of $\operatorname{Af} \rho$ as a function of projected cometocentric distance $\rho$ and of $r_{\mathrm{h}}$.

On the other hand, the spectra have provided us with $\mathrm{CN}, \mathrm{C}_{2}$, $\mathrm{C}_{3}$ and $\mathrm{NH}_{2}$ production rates in the frame of the Haser modelling (Haser 1957), and with dust brightness profiles as a function $\rho$ in the Sun-antisunward direction.

\section{Data analysis and results}

\subsection{Morphology of coma structures}

To determine whether some morphological structures are present in the coma $67 \mathrm{P} /$ Churyumov-Gerasimenko, we enhanced the $R$ filter calibrated images using three different methods: (1) adaptive Laplace filtering as described in Boehnhardt \& Birkle (1994) and references therein; (2) radial normalization (A'Hearn et al. 1986) for verification in case there are features after the Laplace filtering; and (3) Sekanina-Larson method (Larson \& Sekanina 1984). An independent verification was also done by looking for anisotropies in the isophotes. For this study, we used a sequence of 21 images depicting the evolution of the coma between January 25 and April 16, 2009. This analysis was done on images acquired either under photometric or non-photometric sky conditions as the coma morphology does not depend on them.
The description of coma phenomena is based upon what we found in the Laplace filtered images, and in the Sekanina-Larson processed images, and verified as much as possible in the radially normalized images and in the isophote images

From January 25 to 31, the isophote images show a distorted coma: there is a the long spike in the SW direction (presumably the dust trail) as well as are indications of two more structures, one pointing in approximately the Sun direction, and another at $\mathrm{PA} \sim 330^{\circ}$. Both of them become clear after applying any enhancement technique. Figure 1 shows the observed comet image on January 30 after reduction, the Laplace filtered image, and the same image after applying the radial renormalization enhancement technique

During February, the dust coma evolves, the isophotes become more distorted (see Fig. 2), and the structures that are barely seen in January can be undoubtedly detected. On February 18, we can determine three structures at position angles (measured counterclockwise from the north) PA of $40^{\circ}$ slightly deviated from the Sun direction, of $115^{\circ}$, and of $170^{\circ}$. A very detailed inspection of the Laplace filtered image in Fig. 2 allows us to infer a fourth short structure, rather close to the dust tail, at $\mathrm{PA} \sim 225^{\circ}$. Beside these jets, the long spike in the SW direction is notably enhanced by any of the methods we have used.

Later on, the broad structure at PA $40^{\circ}$ seems to split into two narrower ones on February 26, to become only one and much fainter during March. This evolution can be seen in Fig. 3. 

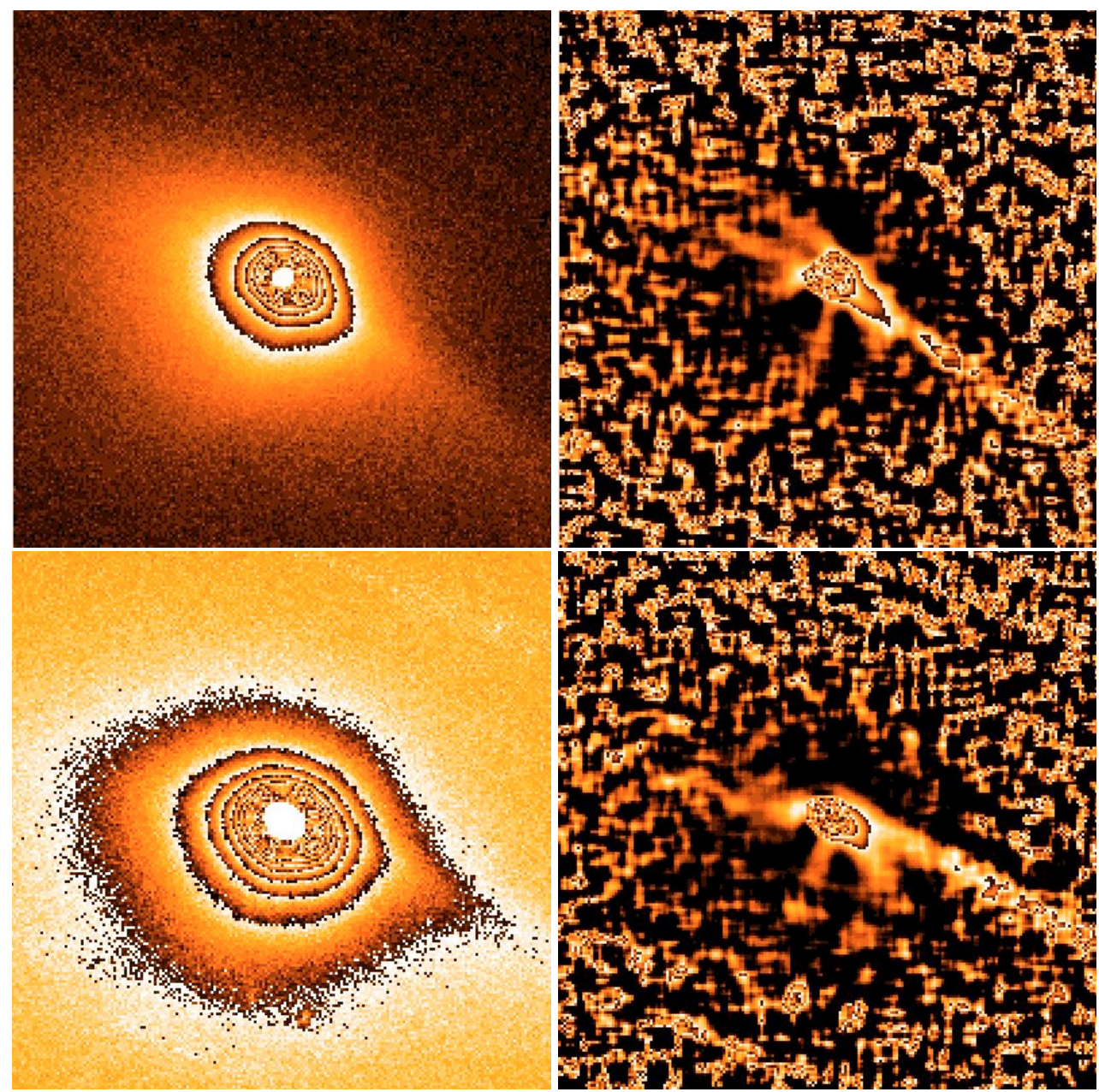

Fig. 3. Evolution of the inner dust coma isophotes and structures of comet 67P/Churyumov-Gerasimenko in 3 weeks. Top row: Feb. 26. Bottom row: March 19. The FOV is $106^{\prime \prime} \times 106^{\prime \prime}$ (i.e. $129300 \times 129300 \mathrm{~km}$ and $133100 \times 133100 \mathrm{~km}$ ), $\mathrm{N}$ is up and E to the left. The two structures on Feb. 26 in the NE quadrant only became one and much fainter fainter on March 19, and is slightly curved and is placed at $\sim 75^{\circ}$ counterclockwise from the north.

Additionally, the faint structure at $\sim 300^{\circ}$ visible during January and February disappears in the images of March 12 and 19, whereas on those dates the fourth structure detected in the January and February images have moved more to the south and are now clearly distinguishable from the dust tail.

A more detailed study of the evolution of the dust coma structures, retrieval of the spin axis orientation, and determination of the number and location of active areas will be presented in a forthcoming paper (Vincent et al. 2011, in prep.). Neither Sun-comet-observer angle (i.e. phase angle) nor the position angles of the extended Sun-comet radius vector ("PsAng") has noticeably changed in the period of time covered by our observations, therefore the changes in the dust coma morphology are not due to a different viewing geometry, but intrinsic to the variability in the cometary dust coma.

Beside these structures, i.e. straight jets, the radially renormalized images also show a clear asymmetry in the Sunantisunward hemisphere, the sunward hemisphere depicting a higher brightness in $R$ Johnson filter for every date that we have monitored the comet (see Fig. 4).

The comet also displayed structures in its dust coma at the $1995 / 96$ apparition, whereas during the 1982/83 apparition no available publications on dust coma morphology were found. In fact, Osip et al. (1992), who analysed data pertaining to the $1982 / 83$ perihelion passage, only mention an asymmetry about perihelion in all species with peak productivity occurring in the month following the perihelion. Schleicher (2006) obtained a limited number of images of comet $67 \mathrm{P} / \mathrm{C}-\mathrm{G}$ on January 25, 1996, one week after perihelion. The images were enhanced by removing a $1 / \rho$ profile and they detected a strong feature towards the southwest, at a position angle (PA) of about $230-240^{\circ}$, linking this structure with a jet apparently emitted from somewhere on the sunward hemisphere of the nucleus. A second more diffuse feature extends towards the east and is centered at PA of $85^{\circ}$ linked to older material within a dust tail. They also reported a general asymmetry with more material towards the southeast than to the northwest. Basically, the structures described by Schleicher (2006) broadly correspond to the ones described in this paper pertaining to the 2009 perihelion passage. However, Schleicher (2006) did not find any additional feature to those mentioned above, whereas our analysis indeed indicates narrow and faint structures at several position angles in the coma whose evolution with time during the 4 months monitoring results in changes of the shape, curvature and number. During March 2003 when the comet was moving outbound from $r_{\mathrm{h}} 2.47$ to $3.06 \mathrm{AU}$, Weiler et al. (2004) obtained a series of images in $R$-broadband filter in which an asymmetric coma and an extended neck-line structure could be seen (since the Earth was very close to the comet's orbital plane). In addition to this, two more structures at PA of $\sim 125^{\circ}$ (tentatively identified as part of 

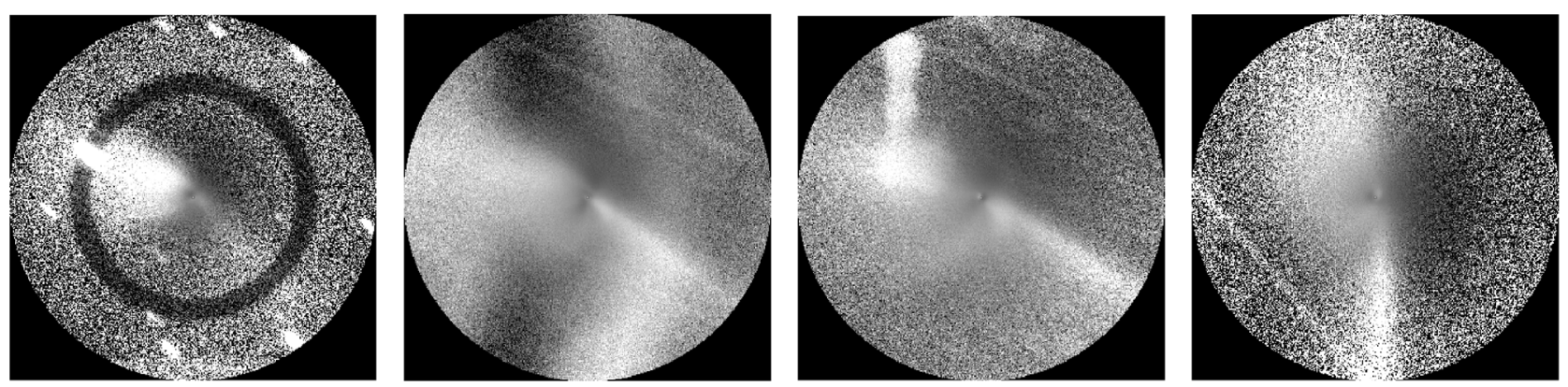

Fig. 4. Radially renormalized images of 67P/Churyumov-Gerasimenko acquired with $R$ Johnson filter on the following dates (from left to right): January 25, February 18, March 12, and April 11, 2009. North is up and east to the left. The FOV is $90^{\prime \prime} \times 90^{\prime \prime}$ for every frame, resulting into $85600 \times 85600 \mathrm{~km}, 81700 \times 81700 \mathrm{~km}, 81900 \times 81900 \mathrm{~km}$, and $88000 \times 88000 \mathrm{~km}$, respectively.

the tail structure) and $\sim 200^{\circ}$ were detected. From February 20 to April 20, 2003, Lara et al. (2005) also analysed a series of broadband images of comet $67 \mathrm{P} / \mathrm{C}-\mathrm{G}$ and arrived at the same conclusion, that is, there is asymmetric dust coma, a bright, thin structure at $\sim 294^{\circ}$, and two further jet-like features at position angles $\sim 95^{\circ}$ and $\sim 195^{\circ}$. Thus, from the complete available set of observations since 1982 , we can conclude the comet $67 \mathrm{P} / \mathrm{C}$ $\mathrm{G}$ does not show a symmetric dust coma, but a dust coma with structures in it whose size, shape, and number change with the heliocentric distance and with viewing geometry.

\subsection{Dust: Af $\rho$ and radial profiles}

The release of dust from the comet is approximately determined through optical measurements of the parameter $\operatorname{Af} \rho(\mathrm{cm})$ as a function of the projected cometocentric distance $\rho$ (A'Hearn et al. 1984)

$\operatorname{Af} \rho=\frac{\left(2 \Delta r_{\mathrm{h}}\right)^{2}}{\rho} \frac{F_{\mathrm{c}}}{F_{\mathrm{S}}}$

where $\Delta$ (in $\mathrm{cm}$ ) and $r_{\mathrm{h}}$ (in $\mathrm{AU}$ ) are the comet's geocentric and heliocentric distances, respectively, $F_{\mathrm{c}}$ is the measured cometary flux in the selected filter ( $R$ Johnson in our case), integrated in the radius of aperture $\rho$, and $F_{\mathrm{S}}$ is the total solar flux in the same filter.

Table 1 contains the Af $\rho$ values we were able to measure during our campaign. There is a clear increase when the comet approaches the perihelion on February 28, 2009. Unfortunately, this date is not covered by our observations. As the phase angle barely changes by $2^{\circ}$ during our observations, the measured variations in the Af $\rho$ parameter do clearly reflect the evolution in dust activity, because the effect of phase angle is not responsible for these variations. Our photometric observation campaign was resumed on March 12, when the $\operatorname{Af} \rho$ values are very similar to the ones during February 25 and 26 (shortly before the perihelion); therefore, it is impossible to conclude whether the peak dust activity took place close to the perihelion date or afterwards. Compared with previous passages, the Af $\rho$ is rather similar to the measurements by Osip et al. (1992) $(\log ($ Af $\rho$ ) between 2.4 and 2.6 to be compared with Fig. 1 in both Osip et al. 1992; Schleicher 2006), and thus slightly higher than the values reported by Schleicher (2006) who claimed an overall decrease in derived Af $\rho$ values during the 1995/96 apparition compared to 1982/83. Schleicher (2006) explains those differences between 1982/83 and 1995/96 passages by their different aperture sizes (i.e. larger in the case of the 1995/96 data set) for computing the cometary flux and not to real evolutionary effects. The Af $\rho$ values listed in Table 1 were computed within apertures of radius equal $10000 \mathrm{~km}$ at the comet distance, that is, an angular diameter aperture of $\sim 16^{\prime \prime} .2$, which is in line with the small aperture sizes used by Schleicher (2006) for computing Af $\rho$ during the $1982 / 83$ passage.

The behaviour of the continuum intensity, $F$ (flux energy), versus $\rho$ was studied from the azimuthally averaged profiles of every comet image acquired in $R$ broad-band Johnson filter regardless the sky conditions excluding those with low $\mathrm{S} / \mathrm{N}$ due to passing clouds. The brightness intensity $F$ as a function of the projected cometocentric distance can be fit with a line if expressed in log-log representation. Least-square fits were computed in two different ranges $1^{\prime \prime}<\rho \leq 90^{\prime \prime}$ and $1^{\prime \prime}<\rho \leq 20^{\prime \prime}$, where the lower limit was selected as it is the mean seeing disk in our observations, whereas the upper limit is the distance at which clear comet signal is still well above the sky background for determining $m_{1}$, and the inner coma a for determining $m_{2}$. The values of the slopes of these fits, $m_{1,2}$ are $-1.58 \leq m \leq-1.09$ between January 25 and March 19 (see Table 1) where the effect of the radiation pressure is clearly seen, giving rise to steeper profiles when considering greater cometocentric distances $\rho$. The determination of $m$ is characterized by rms errors that are always lower than $5 \%$.

A more thorough study of the dust radial profiles was done by determining the intensity versus $\rho$ at position angles where the structures are clearly visible. For this, we converted the images into polar coordinates and located the angular positions where deviations from a spherical dust coma are more noticeable. For February 23 and March 12, these angles are at about $80^{\circ}$ and $\sim 230-240^{\circ}$ (see Fig. 5).

Then, the radial profiles $\log F$ versus $\log \rho$ are computed by azimuthally averaging the measured emission in position angles of $(80 \pm 30)^{\circ}$ and $(230 \pm 20)^{\circ}$ for both dates. The results of this investigation can be seen in Figs. 5 and 6. On March 12, the emission at PA $\sim 80^{\circ}$, approximately the Sun direction, was higher than on February 23 even though the comet was receding from the Sun (see also Table 1), whereas the energy intensity at the neck-line angular position is lower after perihelion. Beside this, the slope of the linear fits to $\log F$ versus $\log \rho$ differ from a position angle of $80^{\circ}$ and in the direction of the dust tail as projected on the sky plane $\left(230^{\circ}\right)$. The values of $m$ are -1.50 and -1.57 for Feb. 23 at the azimuthal angles mentioned above, whereas this slope is -1.26 and -1.47 for March 12 at the same angular positions, indicating a higher dust coma anisotropy on March 12 than on Feb. 23.

The spectra also provide us with pure continuum brightness profiles selected from the following spectral ranges: 482-485, $520-525$, and $680-690 \mathrm{~nm}$. The slope of linear fit to these $\log B-\log \rho$ profiles in the range $3000 \leq \rho \leq 63100 \mathrm{~km}$ 


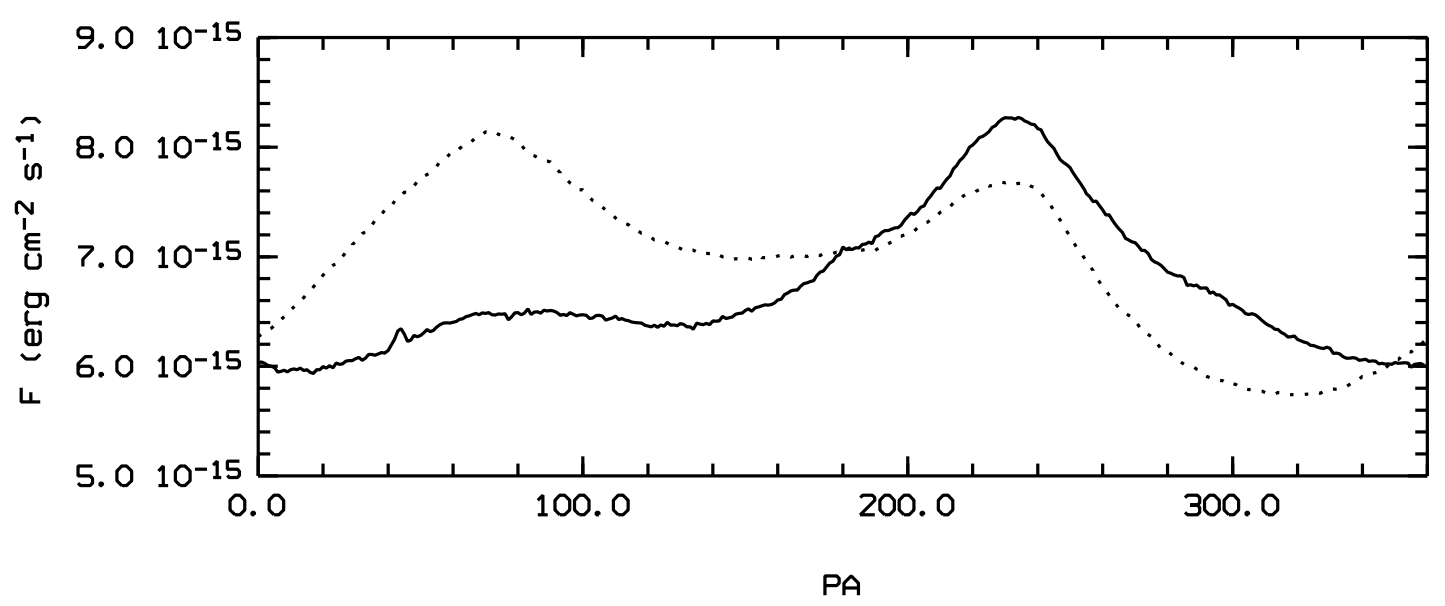

Fig. 5. Azimuthal profile of the brightness intensity for the dust coma of the comet 67P on Feb. 23 (solid line) and March 12 (dashed line) as averaged between the optocentre and 51.5". The change of the dust coma morphology and of the intensity of the structures from almost perihelion to post-perihelion on March 12. The structure at a position angle of $80^{\circ}$ is more pronounced in the post-perihelion than during closest distances to the Sun. On the other hand, the dust tail shows slightly less intensity after the perihelion.

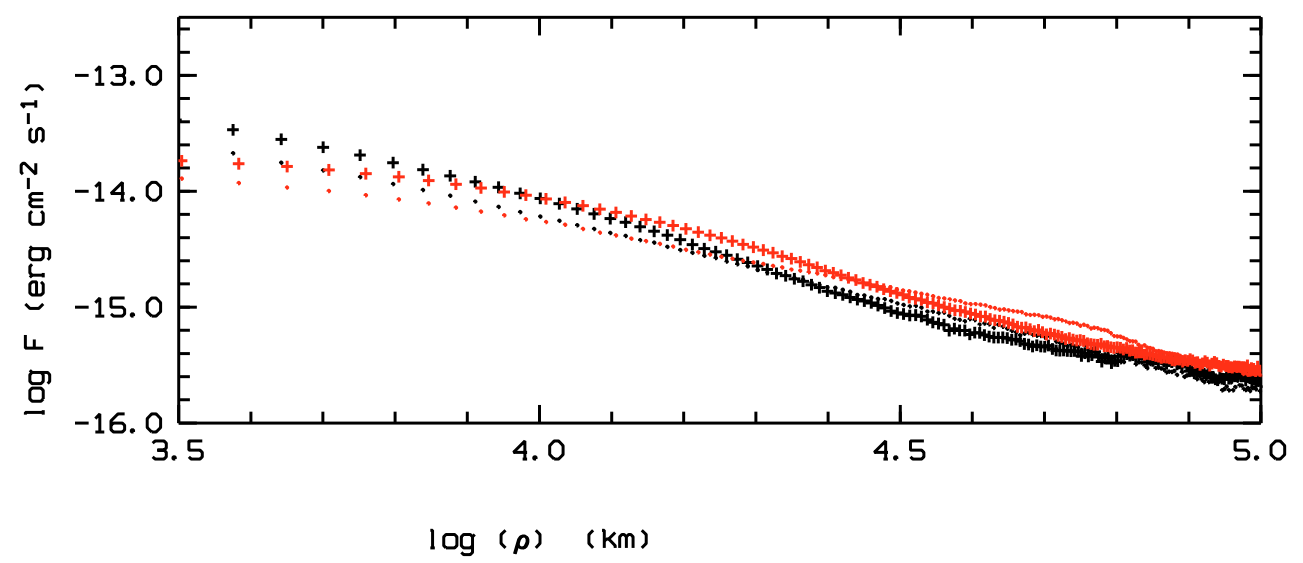

Fig. 6. Dust radial profiles, in $\log F-\log \rho$ representation at position angles $80^{\circ}$ (dots) and $230^{\circ}$ (crosses) for Feb. 23 (in black) and March 12 (in red).

provides the values listed in Table 2 , which are $m \approx-1$. An example of those profiles on Feb. 23 and March 19 are shown in Fig. 7, together with the best linear fit. At large projected cometocentric distances, the $\mathrm{S} / \mathrm{N}$ in the spectrophotometric observations is not good enough to obtain a meaningful fit at those distances. In this regard, the values of $m_{1}$ listed in Table 1 are more reliable when considering the dust coma up to $100000 \mathrm{~km}$ from the nucleus. Furthermore, the values obtained from the spectra in Sun's direction are closer to the canonical behaviour of $m \approx-1$ of the inner dust coma of long-lived grains expanding at constant speed, whereas in the antisunward direction, $m$ approaches the values listed in Table 1, which are more typical of dust grains already affected by solar radiation pressure and $m \approx-1.5$. In fact, by selecting cometocentric distances in the range $3200-64000 \mathrm{~km}$ from the images, the slope $m$ of the linear fits in log-log representation in Sun-antisunward direction is in very good agreement with results from the long-slit spectrophotometric data.

\subsection{Gas production rates}

The spectra of the comet are used to investigate the $\mathrm{CN}, \mathrm{C}_{2}, \mathrm{C}_{3}$, and $\mathrm{NH}_{2}$, and dust continuum profiles in the Sun-antisunward direction. This was possible only on three dates around the comet's perihelion (February 22-24) and on March 19. No further spectra were acquired during the monitoring campaign.

The spectral regions and the subtraction of the underlying continuum in the gas emission bands is done as described by (Lara et al. 2001). The conversion of the emission band fluxes into column densities makes use of constant $g$-factors for $\mathrm{C}_{2}$, $\mathrm{C}_{3}$ and $\mathrm{NH}_{2}$ (A'Hearn et al. 1995), whereas the $g$-factor of the $\mathrm{CN}$ molecule is calculated for the heliocentric distance and velocity of $67 \mathrm{P} / \mathrm{C}-\mathrm{G}$ on every date from the set of values given by Schleicher (1983). The gas production rates are obtained by means of the Haser (Haser 1957) modelling with parent velocity $v_{\mathrm{p}}$ scaled with $r_{\mathrm{h}}\left(v_{\mathrm{p}}=0.86 r_{\mathrm{h}}^{-0.4} \mathrm{~km} \mathrm{~s}^{-1}\right)$, customary values for the daughter velocity $v_{\mathrm{d}}=1 \mathrm{~km} \mathrm{~s}^{-1}$ and scale lengths given in A'Hearn et al. (1995). For the corresponding set of parameters in the Haser modelling, we produced theoretical column density profiles for each species by varying the production rate until the best match between observations and theoretical predictions is achieved.

The production rates obtained that better match the observed column densities and $\log \left[Q\left(\mathrm{C}_{2}\right) / Q(\mathrm{CN})\right], \log \left[Q\left(\mathrm{C}_{3}\right) / Q(\mathrm{CN})\right]$ and $\log [\operatorname{Af} \rho / \mathrm{Q}(\mathrm{CN})]$ are listed in Table 3, whereas as an example, Fig. 8 shows the observed $\mathrm{CN}$ and $\mathrm{C}_{3}$ column density radial profiles in both the anti-sunward and sunward directions together with the best achievable Haser fit. 
Table 2. Linear fits to the $\log B-\log \rho$ profiles obtained form the long-slit spectroscopic measurements.

\begin{tabular}{cccccc}
\hline \hline Date & Direction & \multicolumn{3}{c}{ Continuum regions } & \multirow{2}{*}{ Location } \\
2009 & & $482-485 \mathrm{~nm}$ & $520-525$ & $680-690 \mathrm{~nm}$ & \\
\hline February 22 & sunward & -0.93 & -1.03 & -1.10 & CA \\
& antisunward & -0.92 & -1.11 & -1.23 & CA \\
February 23 & sunward & -1.13 & -1.19 & -1.20 & CA \\
& antisunward & -1.23 & -1.34 & -1.36 & CA \\
February 24 & sunward & -0.81 & -0.91 & -1.05 & CA \\
& antisunward & -0.87 & -0.98 & -1.18 & CA \\
March 19 & sunward & -0.96 & -1.09 & -1.27 & CA \\
& antisunward & -1.00 & -1.14 & -1.31 & CA \\
\hline
\end{tabular}

Notes. $m$ : slope of the linear fit $\log B$ vs. $\log \rho$ for $3.0 \leq \log \rho \leq 4.4$ (i.e. $1000 \leq \rho \leq 25000$ ). Standard deviation of these fits is always lower than $5 \%$.

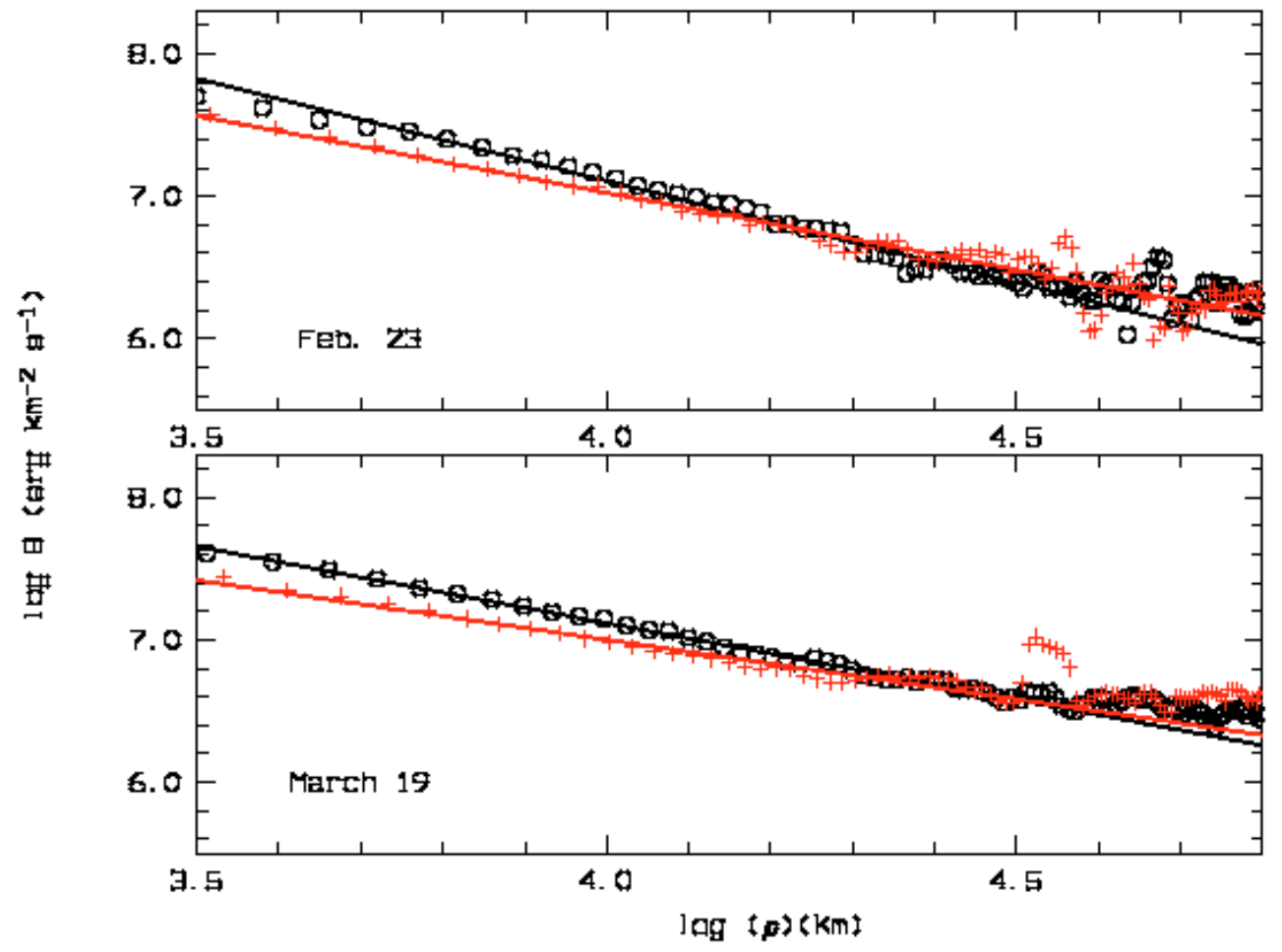

Fig. 7. Dust brightness profiles, in $\log \mathrm{B}-\log \rho$ representation obtained from the spectroscopic measurements on Feb. 23 and March 19, 2009 in Sun (red crosses) - antisunward (black open circles) direction. The selected continuum region is $482-485 \mathrm{~nm}$. Symbols refer to the observational data and solid line refers to the linear fit.

The quotients of the $\mathrm{C}_{2}$ and $\mathrm{CN}$ gas production rates show relatively stable values during the days the spectra were acquired. According to the results listed in Table 3, comet 67P/C-G can be classified as typical following the taxonomic types identified in A'Hearn et al. (1995). The $\mathrm{CN}, \mathrm{C}_{3}$, and $\mathrm{NH}_{2}$ production rates display rather stable values in that short time span decreasing with increasing heliocentric distance. This trend seems to contradict previous passages results in which the peak activity was reached during the month following the perihelion. Our observations only cover 2 weeks after perihelion, and thus, the minimum measured on March 19, 2009 could be only due to short time variations and not a global decrease in comet activity when receding from perihelion.

The quotient $\log [\operatorname{Af} \rho / Q(\mathrm{CN})]$ has a value of $\sim-22$ on the only two dates the quotient could be derived. The $\log [\operatorname{Af} \rho / Q(\mathrm{CN})]$ is in the same order of magnitude as reported in previous apparitions around the perihelion $(\sim-22.3$ from Table 1 in Osip et al. 1992; and Table 3 in Schleicher 2006). In spite of the closer heliocentric distance at perihelion during the 2009 passage, the derived gas production rates are very much in the same order of magnitude as during the 1982 $\left(r_{\mathrm{h}} \sim 1.35 \mathrm{AU}\right)$ and $1995\left(r_{\mathrm{h}} \sim 1.31 \mathrm{AU}\right.$ in 1995) passages (see Schleicher 2006).

\section{Summary}

The ground-based observation campaign of the comet $67 \mathrm{P} / \mathrm{C}-\mathrm{G}$ during its last perihelion passage before the Rosetta s/c encounter has provided us with the most recent information about the comet's activity. During the 4 months (mid-January to midApril 2009) we monitored the comet, we witnessed the development and evolution of several dust structures in the inner coma, as well as the long reported dust trail. The analysis enhancement techniques applied to the whole data set clearly show 3 to 4 structures in the inner dust coma. The evolution of these structures from January to April 2010 is briefly described in this article. A more thorough analysis is being carried out by Vincent et al. (2011, in prep.) and it will allow determination of 
Table 3. Gas production rates 67P/Churyumov-Gerasimenko.

\begin{tabular}{cccccccccc}
\hline \hline Date & $r_{\mathrm{h}}$ & $\Delta$ & $\Delta T$ & $Q(\mathrm{CN})$ & $Q\left(\mathrm{C}_{2}\right)$ & $Q\left(\mathrm{C}_{3}\right)$ & $Q\left(\mathrm{NH}_{2}\right)$ & $\log \left[Q\left(\mathrm{C}_{2}\right) / Q(\mathrm{CN})\right]$ & $\log [\mathrm{Af} \rho / Q(\mathrm{CN})]$ \\
\hline February 22 & 1.247 & 1.678 & -6 & $4.53 \times 10^{24}$ & $3.72 \times 10^{24}$ & $2.82 \times 10^{23}$ & - & -0.09 & - \\
February 23 & 1.247 & 1.678 & -5 & $6.68 \times 10^{24}$ & $5.85 \times 10^{24}$ & $6.19 \times 10^{23}$ & - & -0.12 & -22.29 \\
February 24 & 1.247 & 1.679 & -4 & $6.69 \times 10^{24}$ & $5.42 \times 10^{24}$ & $5.96 \times 10^{23}$ & $1.39 \times 10^{26}$ & -0.09 & - \\
March 19 & 1.269 & 1.732 & +19 & $4.97 \times 10^{24}$ & - & $2.18 \times 10^{23}$ & $1.09 \times 10^{26}$ & - & -22.11 \\
\hline
\end{tabular}
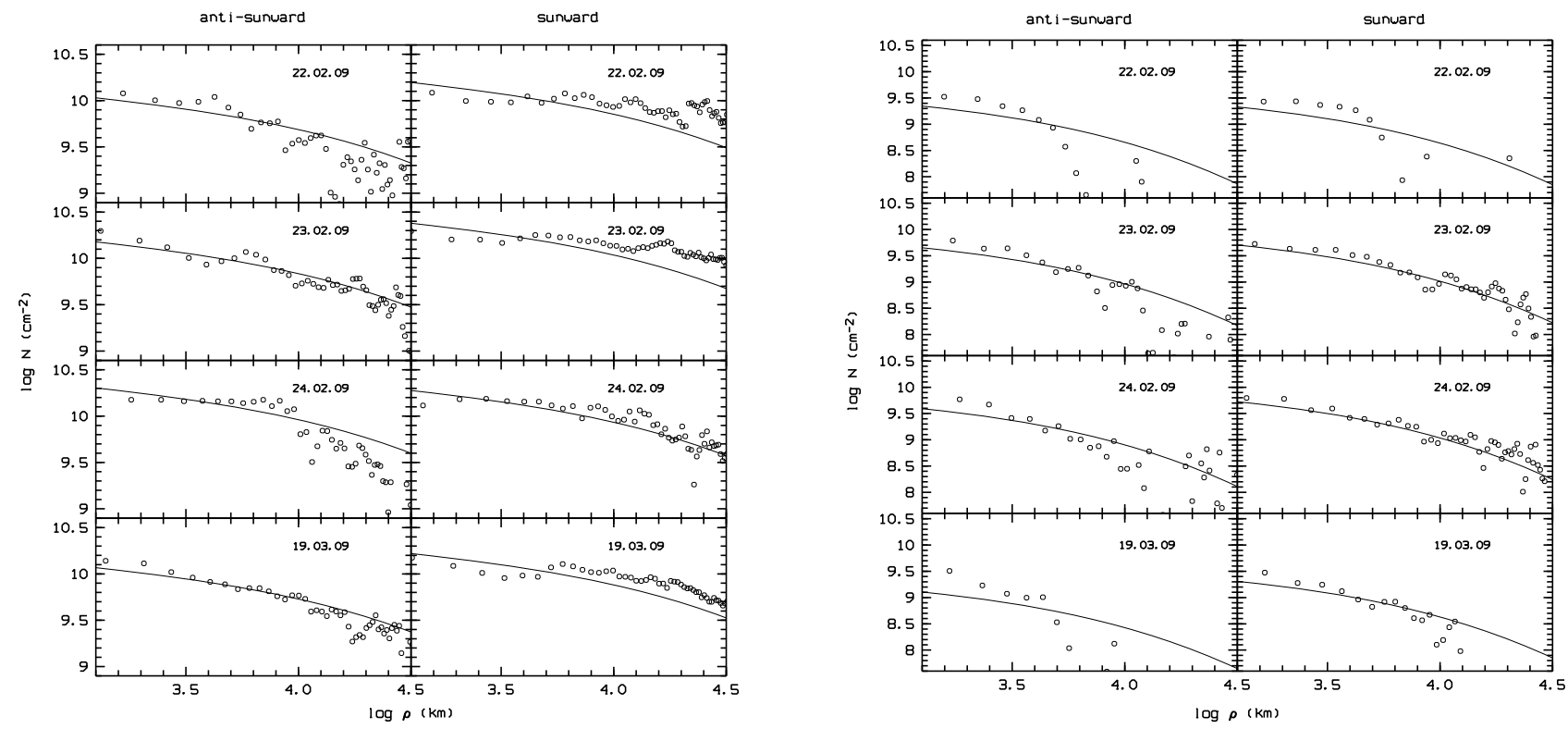

Fig. 8. Observed $\mathrm{CN}$ and $\mathrm{C}_{3}$ column density profiles in $\mathrm{cm}^{-2}$ versus $\rho$ (open circles) in log-log representation together with the best achievable fit (solid line) obtained by means of the Haser modelling (Haser 1957) with parameters given in the text and production rates listed in Table 3.

the rotational state, as well as of the location and number of the active areas on the nucleus.

Both the structures in the dust coma and the Af $\rho$ parameter, as a proxy of the comet dust activity, show a clear evolution between 1.314 AU pre-perithelion and 1.269 AU post-perihelion. Our dataset allows us to derive a (most likely relative) maximum dust activity, determined through Af $\rho$ takes place on March 12, that is, 12 days after perihelion. From an exhaustive analysis of the reported observations during 1982, 1996 and 2002 (Osip et al. 1992; Weiler et al. 2004; Lara et al. 2005; Schleicher 2006), de Almeida et al. (2009) note that 67P/C-G displays an asymmetry in water (or gas) release rates close to perihelion with peak productivity about two months after perihelion. Unfortunately, we monitored the comet until March 19, barely 3 weeks after perihelion, so we cannot confirm that the comet indeed peaks activity two months after the 2009 perihelion on February 28. Our dataset clearly indicates that the dust activity, determined through Af $\rho$, peaked on March 12. However, we cannot conclude whether this maximum is absolute or part of the increasing activity towards a maximum at about two months after perihelion.

The production rates of $\mathrm{CN}, \mathrm{C}_{3}, \mathrm{C}_{2}$, and $\mathrm{NH}_{2}$ are in the same order of magnitude as during previous apparitions. The closer perihelion distance in 2009 (1.24 AU vs. 1.30 AU in 1982 and 1995) has not induced important changes in the gas sublimation rate. It must be noted that the production rate of the above-mentioned species shortly before the perihelion and about 3 weeks after perihelion is rather similar. This result contrasts with the values of Af $\rho$ between February 23 and March 19 when a clear increase in the dust activity occurs, which is not the case for the production rate of gaseous species such as $\mathrm{CN}, \mathrm{C}_{3}, \mathrm{C}_{2}$ and $\mathrm{NH}_{2}$.
Acknowledgements. The paper is based on observations collected at the Centro Astronómico Hispano Alemán (CAHA) at Calar Alto, operated jointly by the Max-Planck Institut für Astronomie and the Instituto de Astrofisica de Andalucia (CSIC). We are grateful to the service observers J. Aceituno, A. Aguirre, M. Alises, A. Guijarro, and F. Hoyo. Without their engagement and dedication, this programme would not have been successful. The research carried out has been partially supported by the Spanish Ministerio de Educación y Ciencia under contract ESP2006-02934 and by the Ministerio de Ciencia e Innovación under contract AyA 2009-08011. Z.-Y. Lin acknowledges a grant by the Junta de Andalucia under the project JA2007-TIC2744. The work of W.-H. Ip is supported by NSC 97-2112-M-008-001-MY3.

\section{References}

A'Hearn, M. F., Schleicher, D. G., Millis, R. L., Feldman, P. D., \& Thompson, D. T. 1984, AJ, 89, 579

A'Hearn, M. F., Hoban, S., Birch, P. V., et al. 1986, Nature, 324, 649

A'Hearn, M. F., Millis, R. L., Schleicher, D. G., Osip, D. J., \& Birch, P. V. 1995, Icarus, 118, 223

Bertini, I., Lara, L. M., Vincent, J., et al. 2009, A\&A, 496, 235

Boehnhardt, H., \& Birkle, K. 1994, A\&AS, 107, 101

de Almeida, A. A., Trevisan Sanzovo, D., Sanzovo, G. C., Boczko, R., \& Miguel Torres, R. 2009, Adv. Space Res., 43, 1993

Ferrín, I. 2010, Planet. Space Sci., 58, 365

Haser, L. 1957, Bull. Soc. R. Sci. Liege, 43, 740

Lara, L. M., Schulz, R., Stüwe, J. A., \& Tozzi, G. P. 2001, Icarus, 150, 124

Lara, L. M., de León, J., Licandro, J., \& Gutiérrez, P. J. 2005, Earth Moon and Planets, 97, 165

Larson, S. M., \& Sekanina, Z. 1984, AJ, 89, 571

Lin, Z., Lin, C., Ip, W., \& Lara, L. M. 2009, AJ, 138, 625

Osip, D. J., Schleicher, D. G., \& Millis, R. L. 1992, Icarus, 98, 115

Schleicher, D. G. 1983, Ph.D. Thesis, AA, Maryland Univ., College Park

Schleicher, D. G. 2006, Icarus, 181, 442

Weiler, M., Rauer, H., \& Helbert, J. 2004, A\&A, 414, 749 